Methods An action research approach using two focus groups (Health Care Professionals $(\mathrm{N}=7)$ and TYA with cancer $(\mathrm{N}=7)$ and an online questionnaire (YSC's $\mathrm{N}=28$ ) was used. Thematic analysis ensued. A research steering group engaging YSC's and Lead Nurses ensured consistency with the methodology and helped shape findings.

Results The positive value of YSC contribution to patients and MDT was echoed consistently across the data. Four domains of practice emerged for inclusion in the framework - 1. Adolescent development 2. The Teenager/Young Adult with Cancer 3.Working with TYA with cancer and 4. Professional practice of YSC work. Discussion and

Conclusions Understanding adolescent development from biopsycho social perspectives, alongside how cancer and its treatment impacts this, are two fundamental domains of practice in YSC work. Youth focused activities is pivotal to the third domain as it creates space to focus on the unique psycho-social needs of AYA with cancer in clinical and nonclinical settings. Professional practice as a domain focusses on the cultures, rules and practices of working in healthcare systems alongside representing and responding to their funder. Further questions and challenges are raised; e.g., the value and challenge of holding purposeful therapeutic conversations; the provision of practice supervision and guidance for YSC whose 'insider/outsider' perspective brings additional practice complexity. It is suggested that these practice insights may not unique to TYA cancer care, and that the findings could have implications for other areas of adolescent healthcare provision.

\section{P66 TRANSITIONING ADOLESCENTS AND YOUNG ADULTS LIVING WITH HIV TO ADULT CARE}

N Desai ${ }^{*}$, J Carlucci, K Garguilo, G Wilson. Pediatrics, Vanderbilt University Medical Center, Nashville, USA

\subsection{6/bmjpo-2019-RCPCH-SAHM.68}

Background Adolescents and young adults living with HIV (AYALWH) comprise a heterogeneous population but have a common need for successful transitioning to adult care. Many institutions have formal transitioning protocols but many obstacles to successful implementation exist.

Aims The purpose of this project was to investigate, design, and implement a Transition Clinic for AYALWH with a goal of maximizing retention during the transition and addressing their comprehensive health care needs.

Methods We initially conducted a literature review to identify best practices of engaging AYALWH to care and to identify gaps in health care needs. We then conducted several site visits to learn how other institutions prepared and engaged AYALWH for transition of care. We identified 15 AYALWH (Age 17-26) in our community who were experiencing difficulties in transitions to adult care and had unmet health care needs.

Results We determined the barriers to care for AYALWH in our community are stigma, transportation, and insurance navigation. In August of 2018 we initiated the Adolescent and Young Adult Health Care Transition Clinic. The clinic is staffed by an Adolescent Physician, an HIV care physician, an Adolescent Social Worker, and an Adult Transition Nurse and is embedded within the General Adolescent Clinic. We have seen 11 clients for a total of 23 visits since inception. We have addressed contraceptive/sexual health needs, primary care needs, behavioral health, and transition preparedness by offering a multidisciplinary approach and focusing on Youth Friendly Services. Our team spends time discussing and addressing barriers to care at each visit.

Conclusions A multidisciplinary clinic can engage and improve access to health care for AYALWH. The potential benefits of this approach include reduction in stigma, improved comprehensive care, and focused time to discuss transition to adult care for youth living with a chronic ailment. We have commenced individualizing transition preparedness for each client through an IRB approved transition survey. Future directions include assessing adherence to treatment through our transition program by measuring serial viral loads during successful transition.

\section{P67 ADOLESCENCE, PREGNANCY, AND OUD: CREATING A CLINICAL DECISION TREE THAT GUIDES PROVIDERS THROUGH MAT FOR A HIGH-RISK OBSTETRICAL POPULATION}

${ }^{1} \mathrm{M}$ Thompson*, ${ }^{2} \mathrm{C}$ Jones. ${ }^{1}$ Obstetrics and Gynecology, University of lowa, lowa City, USA; ${ }^{2}$ Obstetrics and Gynecology, University of Minnesota, Minneapolis, USA

\subsection{6/bmjpo-2019-RCPCH-SAHM.69}

Opioid use in pregnancy is on the rise in the United States. There is limited data regarding management for pregnant adolescents with opioid use disorder (OUD). However, there are best practice recommendations for management of OUD in pregnancy that has been demonstrated to improve maternal, fetal, and neonatal outcomes.

Aim The aim of this presentation is to highlight the demographics of pregnant adolescents with OUD, discuss risk and protective factors, and introduce a clinical decision tree for management of OUD in the inpatient obstetrical setting. According to SAMHSA guidelines, adolescents who are pregnant are more likely than their adult counterparts to have past month opioid use. Many inpatient obstetrical providers do not feel comfortable managing pregnant adolescents with OUD and need additional guidance to provide care according to best practice recommendations.

Methods A literature review has been completed regarding adolescents, pregnancy, and OUD. A clinical decision tree was created by a DNP student and a MFM mentor for an inpatient setting and listeners will be guided through continuation or management of MAT for pregnant adolescents with OUD.

Results The clinical decision tree will be reviewed during the presentation.

Conclusion Knowledge regarding adolescents, OUD, and pregnancy can help adolescent health and obstetrical providers improve maternal, fetal, and neonatal outcomes that are linked to lifelong improvementin health outcomees for both mothers and infants. 